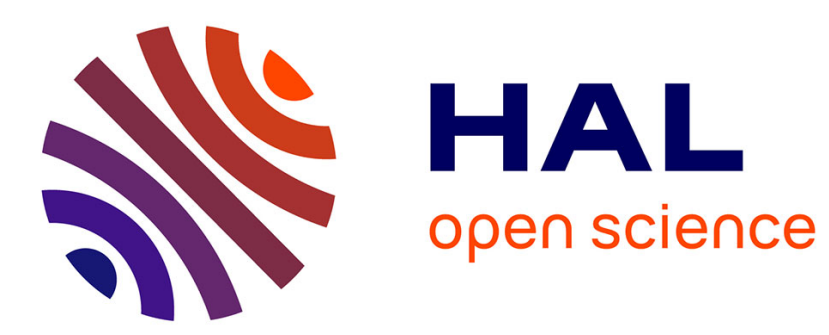

\title{
How to shake the Invisible Hand (when Robinson meets Friday)
}

Antoine Billot

\section{To cite this version:}

Antoine Billot. How to shake the Invisible Hand (when Robinson meets Friday). 2007. halshs00588081

\section{HAL Id: halshs-00588081 https://shs.hal.science/halshs-00588081}

Preprint submitted on 22 Apr 2011

HAL is a multi-disciplinary open access archive for the deposit and dissemination of scientific research documents, whether they are published or not. The documents may come from teaching and research institutions in France or abroad, or from public or private research centers.
L'archive ouverte pluridisciplinaire HAL, est destinée au dépôt et à la diffusion de documents scientifiques de niveau recherche, publiés ou non, émanant des établissements d'enseignement et de recherche français ou étrangers, des laboratoires publics ou privés. 


\section{PARIS SCHOOL OF ECONOMICS}

WORKING PAPER N² 2007 - 13

How to shake the Invisible Hand

(when Robinson meets Friday)

Antoine Billot

JEL Codes: A13, D71, J15

Keywords: preferences, social interaction, invisible hand

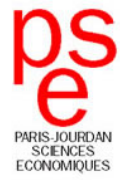




\title{
How to Shake the Invisible Hand (When Robinson Meets Friday)
}

\author{
Antoine Billot* \\ Université Paris 2 Panthéon-Assas and Ecole d'Economie de Paris
}

May 14, 2007

\begin{abstract}
We propose to define the invisible hand by (i) modelling the mechanism itself (not to just assume its existence) and (ii) making explicit the limit conditions for its working. For that purpose, we simply assimilate the working of the invisible hand mechanism to the existence of a social preference such that individual and social optimalities are consistent. In introducing the possibility of interaction among individuals, we then suggest that the standard 'Robinson case' or social atomicity is just a degenerate feature of a more general requirement that we call the Global Network Agreement. Our main result is that the invisible hand mechanism does keep on working when there is an interaction between Robinson and Friday if the former (resp. the latter) is sensitive to the latter (resp. the former) in such a way that they exhibit some agreement in preferences. Hence, the 'Robinson case' naturally satisfies this property since nor Robinson neither Friday can disagree with himself. But more cooperative situations are also allowed in order to extent the invisible hand mechanism to cases with interactions.
\end{abstract}

Nous proposons de définir la Main Invisible sous la forme d'un mécanisme formel explicite. Pour cela, nous assimilons ce mécanisme à l'existence d'une relation de préférence sociale telle que les optimalités individuelle et collective soient compatibles. Puis en introduisant une possibilité d'interaction entre agents, nous suggérons que le cas standard (Adam Smith) d'atomicité sociale' constitue le cas limite d'une classe plus générale de situations satisfaisant toutes une certaine contrainte appelée le "Global Network Agreement" (ou Accord Global de Réseau). Notre résultat principal est alors que le mécanisme de la main invisible s'applique même en présence d'interactions dès lors que les agents qui interagissent sont sensibles en préférence les uns aux autres d'une manière telle qu'ils manifestent entre eux un consensus minimal. Aussi, le cas standard (chaque agent est un Robinson) satisfait naturellement cette propriété,

*I thank Maurice Salles, Jacques-François Thisse, James Friedman, Claudio Mezzetti, the participants of the doctoral seminars at Venice International University and two referees for comments and suggestions. Furthermore, I specially thank Tzachi Gilboa whose remarks clearly improve the proof and even the meaning of the second theorem. Usual disclaimer applies. For correspondence: Université Panthéon-Assas, Paris 2, 92 rue d'Assas, 75006, Paris, France, e-mail address : billot@u-paris2.fr 
cependant, d'autres cas, beaucoup plus coopératifs sont eux aussi aussi compatibles avec le mécanisme de la main invisible.

JEL Classification: A13-D71-J15

Keywords: Preferences, social interaction, invisible hand

\section{Introduction}

The idea that vice or selfishness leads to virtue or social good appears in 1723 with La Fable des Abeilles or Les Vices Privés Font le Bien Public proposed by Bernard Mandeville. ${ }^{1}$ According to Mandeville, a hive is analogous to a human society. And what do we observe in a hive? Two things. First, the bees seem to behave in a coordinated and cooperative way while in reality they are just selfish maximizers of their own (private) welfare. Second, such decentralized actions of the individual bees promote the hive welfare better than could be achieved by a 'central planner bee'. How is it possible? In his famous book on The Wealth of Nations written in 1776, Adam Smith answered: because of the so-called 'invisible hand'. Two centuries later, the invisible hand metaphor still works: the liberal heirs of the classical economists can affirm that markets would spontaneously produce socially optimal results if only government refrains from interference. Yet, such a use of the Mandeville-Smith's intuition is purely ideological: it is a defence for laissez-faire capitalism. (The interpretation of the concept of the invisible hand is definitely subtle and consequently ambiguous: see Nozick, 1981, or Rothschild, 1994, for further discussions). The existence and the proper working of the invisible hand mechanism are taken for granted, without specifying the limit conditions for it to work. In addition, how should one precisely characterize an invisible hand mechanism? First, the outcome to be explained must be produced as a result of a decentralized process: there are no explicit agreements among the individuals. Second, the invisible hand mechanism must be non-intentional: the individuals do not intend to produce the outcome, that is social welfare; a by-product of their actions is the social desirability of the final outcome. Actually, since the process should work even if the individuals have no knowledge of the process, it is called invisible. ${ }^{2}$

In this paper, we propose to define the invisible hand mechanism in a more systematic methodological way. Specifically, we implicitly consider that it is contingent if the mechanism is actually working. What is interesting is rather (i) to model the mechanism itself, not to just assume its existence and (ii) to make explicit the limit conditions for it to work.

For that purpose, recall that an individual optimal decision is a maximal element of an individual preference order and a social optimal decision is a maximal element of a social preference order. Then, we suggest that an invisible hand mechanism naturally works when there is no conflict between each individual optimal decision and the social optimal one. Consequently, we consider

\footnotetext{
${ }^{1}$ Actually, the first version of this text is a long poem published in 1705 under the title: The Grumbling Hive or Knaves Turned Honest.

${ }^{2}$ However, the invisibility is not an essential requirement for a mechanism to be considered as an invisible hand mechanism. The result in question needs not be unknown to the individuals participating in its production. Modern discussion about the invisible hand allows the individuals to know the result of their actions. It is nevertheless required that they do not intend to produce that result. The result should be an unintended consequence of the action, that is a by-product of intentions to do something else.
} 
the working of an invisible hand mechanism as the existence of a social preference for which individual and social optimalities are consistent. The formal conditions of existence of such a social preference characterize the environment for the invisible hand mechanism to work.

Traditionally, one thinks that the invisible hand mechanism needs social atomicity, namely the fact that no individual can influence the social decision by means of his only preferences. However, in introducing interactions among individuals (à la Akerlof, 1997), we suggest that social atomicity, that is the 'Robinson case', is a particular feature of a more general requirement that we call Global Network Agreement: the invisible hand mechanism requires that individuals agree in some sense in preferences when they interact. In other words, each Robinson naturally satisfies this property since no one can disagree with himself. But other situations, definitely more cooperative, are allowed as well.

The organization of the paper is as follows: In Section 2, we present the model of interaction and the utility representation of individual preferences translating interactions (Theorem 1). In Section 3, we model the invisible hand mechanism and prove the limit condition of its working, namely the presence of a local network disagreement (Theorem 2). Finally, in Section 4, we illustrate our result with two examples where the invisible hand mechanism works: first, the Robinson case (no interaction) and second, an Interaction case with Global Network Agreement.

\section{Individuals}

\subsection{Individual Preferences}

Consider a given and finite set $S$ of individuals $i(i: 1, \ldots, n)$. Call coalition any nonempty subset of $S$.

Consider an arbitrary given set $C \subset \mathbb{R}$ of (monetary) consequences expressed by real numbers and $X$ a set of acts, that is functions $x$ from the set $S$ of individuals to that $C$ of consequences. Any act $x$ of $X$ is such that $x(i)$ corresponds to the consequence of the act $x$ for individual $i$.

For any coalition $A \subset S, x(A)$ denotes the tuple $[x(i)]_{i \in A}$. Furthermore, given a coalition $A$ and two acts $x$ and $y$, an act denoted $\left(x_{A}, y_{-A}\right)$ is such that $\left(x_{A}, y_{-A}\right)(i)=x(i)$ for all individuals $i \in A$ and $\left(x_{A}, y_{-A}\right)(j)=y(j)$ for all other individuals $j \notin A$. By convention, we write (i) $x \geq y$ if $x(i) \geq y(i)$ for all individuals $i \in S$, (ii) $x>y$ if $x \geq y$ and $x(i)>y(i)$ for some individuals $i \in S$ and (iii) $x \gg y$ if $x(i)>y(i)$ for all individuals $i \in S$.

Each individual $i \in S$ is characterized by a preference relation $\succcurlyeq_{i}$, that is a binary relation defined over $X \times X$ such that $x \succcurlyeq_{i} y$ means that for individual $i, x$ is at least as good as $y$. Two acts $x, y$ are considered indifferent, which is denoted $x \sim_{i} y$, when we simultaneously have $x \succcurlyeq_{i} y$ and $y \succcurlyeq_{i} x$.

Besides, all preference relations are supposed to be (i) nontrivial: for all $i \in S,\left(x_{i}, z_{-i}\right) \succ_{i}\left(y_{i}, z_{-i}\right)$ for some $x, y, z \in X$ and (ii) monotone: for all $i \in S$, if $x(i)>y(i)$, then $\left(x_{i}, z_{-i}\right) \succ_{i}\left(y_{i}, z_{-i}\right)$ for all $z \in X$.

We also assume the following standard axioms.

Axiom 1 (Complete Weak Ordering) : $\forall i \in S$, the preference relation $\succcurlyeq_{i}$ is a complete weak-order on $X^{2}$. 
Axiom 2 (Continuity) : $\forall i \in S, \forall x, y, z \in X$ such that $x \succcurlyeq_{i} y \succcurlyeq_{i} z$, there exists $\alpha \in[0,1]$ such that $\alpha x+(1-\alpha) z \sim_{i} y$.

Axiom 3 (Independence) $: \forall i \in S, \forall x, y, z \in X$ such that $\left.x \succcurlyeq_{i} y, \forall \alpha \in\right] 0,1[$ : $\alpha x+(1-\alpha) z \succcurlyeq_{i} \alpha y+(1-\alpha) z$.

\subsection{Interaction and vNM Expected Utility Representa- tion}

Several economic situations involve basic interactions sometimes called 'network externalities' such as urban congestion, fashion effect, conformism or altruism and so on... The common idea of all these approaches is that individuals are assumed to be interdependent whenever their preferences may be affected by the social neighborhood (see e.g., Postlewaite, 1998, Billot, 2003). Here, if $i$ interacts with $j$ then $i$ may be 'sufficiently sensitive to $j$ ' to broaden his own set of preferences determinants in order to encapsulate the consequence for $j$ of all acts $i$ faces. ${ }^{3}$ For instance, Luttmer (2001) shows that numerous young black lawyers issued from the poor suburb of Chicago finally militate in favor of a fiscal policy that proposes to largely redistribute towards the poor social classes (even if, for that purpose, they will individually pay more taxes) rather than choosing to support a liberal program which maximizes their selfish interests.

\subsubsection{Interaction}

Consider that there exists a binary relation $I$ defined on $S^{2}$ such that ' $i I j$ ' means that ' $i$ interacts with $j$ ' and assume that this relation $I$ is reflexive: $\forall i \in S, i I i$.

Consider now, for any $i \in S$, a probability distribution $p_{i}: 2^{S} \rightarrow[0,1]$ with $\sum_{j \in S} p_{i}(j)=1$ where $p_{i}(j)$ is the probability for $i$ to be sensitive to $j$. Assume that if $i$ has no interaction with $j$, then $p_{i}(j)=0$, i.e. $i$ 's sensitivity to $j$ is null. On contrary, each time $i I j, p_{i}(j)>0$ and $i$ is sensitive to what happens to $j$. (Intuitively, the more $i$ interacts with $j$, the more he is sensitive to him.) Note that since we suppose $I$ to be reflexive, then $p_{i}(i)>0$. Finally, the support of the distribution $p_{i}$ naturally corresponds to $i$ 's individual network, that is the set of all individuals with whom he has an interaction. We denote it $S_{i}$ :

$$
S_{i}=\{j \in S: i I j\}={ }_{\text {def }}\left\{j \in S: p_{i}(j)>0\right\} .
$$

Note that the reflexivity of $I$ can also be written as $\left[\forall i \in S, i \in S_{i}\right] .^{4}$

Two cases occur:

(1) (Ic as Interaction case). There exists at least one individual whose network is nonsingleton:

$[\exists i, j \in S, i \neq j$, s.t. $i I j] \Longleftrightarrow\left[\exists i \in S\right.$ s.t. $\left.p_{i}(i)<1\right] \Longleftrightarrow\left[\exists i \in S\right.$ s.t. $\left.S_{i} \neq\{i\}\right]$.

For convenience, we say in that case that $p_{i}$ is a nonsingleton distribution.

\footnotetext{
${ }^{3}$ This sort of interaction does not suppose any strategic behavior nor coordination: for instance, an individual $i$ can be sensitive to what happens to another individual $j$ without $j$ being sensitive to $i$.

${ }^{4}$ In the language of epistemic logics à la Kripke (1963) (see for instance Hughes and Cresswell, 1989, or Billot and Walliser, 1999), the individual network $S_{i}$ is called a syntactical operator and the distribution $p_{i}$ is its valued semantical counterpart while the society $S$ plays the role of the set of possible worlds.
} 
(2) (Rc as Robinson case). All individual networks are singleton:

$[\forall i \in S, \exists j \in S, i \neq j$, s.t. $i I j] \Longleftrightarrow\left[\forall i \in S, p_{i}(i)=1\right] \Longleftrightarrow\left[\forall i \in S, S_{i}=\{i\}\right]$.

For convenience, we say in that case that any $p_{i}$ is a singleton distribution.

The standard case, namely that which is commonly used in general microeconomics, is obviously given by Rc.

Remark 1 : Transitivity of the interaction $I-i . e . \forall i, j, k \in S,[i I j$ and $j I k] \Rightarrow$ $[i I k]-$, can also be written as $\left[j \in S_{i} \Rightarrow S_{j} \subset S_{i}\right] .^{5}$

\subsection{2 vNM Expected Utility Representation}

Following Morris intuition in $(1997,2000)$, we then propose to translate the behavior of an individual who is sensitive to other individuals through the probability distribution which affects his utilities in a setting à la von NeumannMorgenstern (vNM) where $u_{i}(x(i))$ translates $i$ 's utility when facing the consequence of $x$ for him, namely $x(i)$, while $u_{i}(x(j))$ corresponds to $i$ 's utility when considering the consequence of $x$ for $j$, namely $x(j)$.

Theorem 1 (vNM) : Under $\boldsymbol{A} \mathbf{1}-\boldsymbol{A} 3$, for each individual $i \in S$, there exists a strictly increasing and continuous utility function $u_{i}: X \rightarrow \mathbb{R}$ such that:

$$
x \succcurlyeq_{i} y \Leftrightarrow \sum_{j \in S} p_{i}(j) u_{i}(x(j)) \geq \sum_{j \in S} p_{i}(j) u_{i}(y(j))
$$

where $p_{i}$ is a given probability distribution, $p_{i}: 2^{S} \rightarrow[0,1]$ with $\sum_{j \in S} p_{i}(j)=1$, translating $i$ 's interactions in $S$. Moreover, $u_{i}$ is unique up to a linear transformation.

The proof is strictly equivalent to that of Proposition 5.4 in Kreps (1988, Ch. 5., 46-50).

Therefore each individual preference $\succcurlyeq_{i}$ satisfying A1-A3 has a $v N M$ representation as defined in (2). This way, if $i I j, i$ takes into account - by empathy - the consequence $x(j)$ of $x$ weighted by the degree $p_{i}(j)$ of his sensitivity to $j$. On the contrary, if $i$ does not interact with $j$, the influence of the consequence $x(j)$ in $i$ 's evaluation of $x$ is null.

In the Robinson case, (2) naturally becomes:

$$
x \succcurlyeq_{i} y \Leftrightarrow u_{i}(x(i)) \geq u_{i}(y(i))
$$

since $S_{i}=\{i\}$ (see also Samet and Schmeidler, 2003, for the interpretation of such a constraint as the qualification rule of liberalism).

Throughout the sequel, we consider that each individual preferences has a vNM representation as defined by (2).

\footnotetext{
${ }^{5}$ See Billot and Walliser $(1999, \S 2.4,190-192)$ for details about the way semantical properties can be equivalently interpreted in syntax.
} 


\section{Invisible Hand}

Consider a finite subset $\Pi$ of acts, $\Pi \subset X$, and call it a problem. A problem corresponds to a particular decision which must be made by the society $S$.

For each problem $\Pi \subset X$ and each individual $i \in S$, it can be defined a set of $i$ 's optimal solutions, that is the acts dominating in preferences all other acts that are candidates to solve the problem:

$$
\Pi_{i}=\left\{x \in \Pi: x \succcurlyeq_{i} y, \text { for all } y \in \Pi\right\} .
$$

Proposition 1 : Under $\boldsymbol{A} \mathbf{1}$, each individual optimal solution set $\Pi_{i}$ associated to any problem $\Pi$ is nonempty.

Proof: Straightforward by finiteness of $\Pi$ and weak-ordering completeness of $\succcurlyeq_{i}$

Define now a decision rule as a function $\Phi: S \rightarrow \Pi$ and say $\Phi$ to be optimal when

$$
\forall i \in S, \Phi(i) \in \Pi_{i}
$$

Remark 2 : A decision rule $\Phi$ does take into account the individuals' social network since each $\Pi_{i}$ is based upon i's preference and each i's preference is related to $S_{i}$ by its vNM representation as defined in (2).

When such an optimal decision rule is followed, $\Phi(i)$ determines the acts which dominate - for $i$ - all other possible solutions of the problem. Such a rule generates an abstract new act $\vec{\varphi}$, not necessarily in $\Pi$, such that $\varphi(i)=\Phi_{i}(i)$, $\forall i \in S$ (where $\Phi_{i}(i)$ is the $i$ 's consequence of the act $\Phi(i)$ ). The class of acts generated by optimal decision rules is denoted $\Pi^{*}$ and defined as follows:

$$
\Pi^{*}=\left\{x \in X: x(i)=\Phi_{i}(i), \forall i \in S, \text { for some optimal } \Phi\right\} .
$$

Proposition 2 : Under $\boldsymbol{A} \mathbf{1}-\boldsymbol{A} 3$ the class $\Pi^{*}$ of issues generated by optimal decision rules is nonempty.

Proof: This follows from Proposition 1 and (6)

Each element $x$ of the nonempty class $\Pi^{*}$ of acts generated by optimal decision rules is designed such that the individual consequence $x(i)$ is $\succcurlyeq_{i}$-maximal by definition of $\Pi_{i}$ and (5-6). Then, if there is no social preference such that each act of $\Pi^{*}$ is weakly preferred to any other act of the problem $\Pi$, we know that a conflict arises between the society $S$ as a whole and some of its members: hence the invisible hand does not seem to work. A contrario, if there is no contradiction between the society $S$ as a whole and any of its members in the sense where the best decision for the former is consistent with each best decision of the others, the invisible hand seems to work.

Introduce one supplementary condition on the individual preferences.

Given individual preferences $\left(\succcurlyeq_{i}\right)_{i \in S}$ and a coalition $A \subset S$, say that $x \succcurlyeq_{A} y$ if $x \succcurlyeq_{i} y$ for all $i \in A$, and $x \succ_{A} y$ if $x \succcurlyeq_{A} y$ and $x \succ_{i} y$ for some $i \in A$.

Global Network Agreement : $\forall i \in S, x \succcurlyeq_{i} y \Rightarrow x \succcurlyeq_{S_{i}} y$ for all $x, y \in X$. 
Global Network Agreement means that within each given individual network, no conflict arises between the individual and its own network. In return a local (network) disagreement corresponds to the fact that there exists an individual, say $i$, such that $x \succcurlyeq_{i} y \nRightarrow x \succcurlyeq_{S_{i}} y$ for all $x, y \in X$, i.e. $\exists x^{\prime}, y^{\prime} \in X$ such that $x^{\prime} \succcurlyeq_{i} y^{\prime}$ while $y^{\prime} \succ_{S_{i}} x^{\prime}$.

Lemma 1 : In the Robinson case, there is a Global Network Agreement.

Straigthforward from (Rc).

Note, indeed, that a Global Network Agreement does not imply that $S_{i}=$ $\{i\}, \forall i \in S$. Thus, let us study other situations, especially situations where individual networks are nonsingleton.

Lemma 2 : There is a Global Network Agreement iff the interaction I is transitive.

Proof: $(\Rightarrow)$ Transitivity of $I$ can also be written as $\left[j \in S_{i} \Rightarrow S_{j} \subset S_{i}\right]$ (see Remark 1). It means that, $\forall i, j, k \in S$, if $i \in S_{j}$ and $j \in S_{k}$ then $i \in S_{k}$. Suppose now, on the contrary, that there exist three individuals $1,2,3 \in S$ such that $1 \in S_{2}, 2 \in S_{3}$ but $1 \notin S_{3}$. Let $x(1)=1$ and $x(i)=0$ for all $i \neq 1$. By monotonicity of the preferences, $x \succ_{1} 0_{n}$ (since $1 \in S_{1}$ by reflexivity of $I$ ), $x \succ_{2} 0_{n}$ (since $1 \in S_{2}$ ), $x \sim_{3} 0_{n}$ (since $1 \notin S_{3}$ ) and finally $x \succcurlyeq_{i} 0_{n}$ for all $i \in S$, where $0_{n}=(0, \ldots, 0)$. Thus, $x \succ_{S_{3}} 0_{n}$ since $2 \in S_{3}$. But, $x \sim_{3} 0_{n}$ while $x \succ_{S_{3}} 0_{n}$ means that there is a local disagreement.

$(\Leftarrow)$ Suppose that there is a local disagreement. It means that, for some $i \in S$, $x \succ_{S_{i}} 0_{n}$ while $x \sim_{i} 0_{n}$. Hence, there exists $j \in S_{i}$ such that $x \succ_{j} 0_{n}$. Let $x(k)=1$ and $x(i)=0$ for all $i \neq k$. If $x \succ_{j} 0_{n}$, this means that $k \in S_{j}, k \neq j$, i.e. $\{j, k\} \subset S_{j}$. Since $x \sim_{i} 0_{n}$, then $k \notin S_{i}$. Consequently $j \in S_{i} \nRightarrow S_{j} \subset S_{i}$ and $I$ is not transitive.

Define a social preference $\succcurlyeq^{*}$ as a preference relation which satisfies (A1-A3) and a Strong Monotonicity condition:

$$
\forall i \in S, \forall x, y \in X,\left(x_{i}, z_{-i}\right) \succcurlyeq^{*}\left(y_{i}, z_{-i}\right) \Leftrightarrow x(i) \geq y(i)
$$

This condition translates the fact that, ceteris paribus, the society is better off when allocating more than less to any given individual.

The modeling of an invisible hand mechanism that we propose is then that acts in $\Pi^{*}$ (which may or may not be included in the problem $\Pi$ ) must be at least as good for the social preference $\succcurlyeq^{*}$ as acts in $\Pi$, that is $x \succcurlyeq^{*} y$ for all acts $x \in \Pi^{*}$ and $y \in \Pi$.

Invisible Hand (IH) : There exists a social preference $\succcurlyeq^{*}$ such that for each finite problem $\Pi \subset X$ there exists an act $x \in \Pi^{*}$ such that $x \succcurlyeq^{*} y$, for all $y \in \Pi$.

The Global Network Agreement condition implies that the sensitivity of an individual to another one, when it exists (i.e. in the Interaction case), can be translated through its individual network. Nevertheless, this result does not clarify the implication relation between IH and a Global Network Agreement. 
Does IH implies Global Agreement or the other way round? If it is straightforward that $\mathbf{I H}$ is consistent with the Robinson case, that is when all the individual networks are singleton, we must know if it can be consistent as well with nonsingleton individual networks.

Theorem 2 : If $\mathbf{I H}$ holds, then there is a Global Network Agreement.

Lemma 1 shows that there is a Global Network Agreement iff the interaction $I$ is transitive. Hence we have now to precise the relationship between $\mathbf{I H}$ and the properties of the interaction $I$ :

Lemma 3 : If $\mathbf{I H}$ holds, then the interaction I is transitive.

Proof: Suppose that $I$ does not satisfy transitivity, i.e. $\left[j \in S_{i} \not S_{j} \subset S_{i}\right]$ for some $i, j, k \in S$. This means for instance that there exist three individuals $1,2,3 \in S$ such that $2 \in S_{1}, 1 \in S_{3}$ but $2 \notin S_{3}$. Now, by A2, it is possible to define a problem $\Pi=\{x, 0\}$ where the act $x$ is defined as $\left(\varepsilon_{1},-1,-\varepsilon_{3}, 0, \ldots, 0\right)$ $\left(\right.$ with $x(1)=\varepsilon_{1}, x(2)=-1, x(3)=-\varepsilon_{3}$ and $x(i)=0$ for all $i \neq 1,2,3$ with $\varepsilon_{1}, \varepsilon_{3}$ positive and very small) such that the act $0_{n} \succ_{1} x$ (since $\{1,2\} \subset S_{1}$ and $\varepsilon_{1}$ very small), $0_{n} \succ_{2} x$ (since $2 \in S_{2}$ while $1 \notin S_{2}$ and $3 \notin S_{2}$ ) but $x \succ_{3} 0_{n} .{ }^{6}$ Hence, if $y \in \Pi^{*}$, this implies $y(3)=-\varepsilon_{3}$ and $y(i)=0$ for all $i \neq 3$, i.e. $\Pi^{*}=\{0,0, y(3), 0, \ldots, 0\}$. Thus, $0_{n} \succ^{*} y$ for all $y \in \Pi^{*}$. This is a contradiction with IH.

Proof of Theorem 2: It is straightforward from Lemmas 1 and 2.

If a conflict arises between the society as a whole and some of its members, the invisible hand does not work. This is clearly consistent with a society based on individuals who behave like Robinson. However, Theorem 2 shows that it is possible for the invisible hand mechanism to be relevant whenever 'Robinson meets Friday who meets...' if the interaction between the individuals is transitive such that there is no local disagreement. In that sense, if individuals throught networks are sufficiently connected in preferences, the standard invisible hand mechanism can be extented to subsets of individuals.

\section{Examples}

As an illustration of situations where IH holds, consider the two following examples:

Example 1 (Robinson Case) : Suppose three individuals $\{1,2,3\}$ whose individual distributions are singleton, i.e. $S_{1}=\{1\}, S_{2}=\{2\}$ and $S_{3}=\{3\}$, and utilities are given by $u_{i}(x)=x$ for $i: 1,2,3$. Then, for two programs $x, y$ :

$$
\left\{\begin{array}{l}
x \succcurlyeq_{1} y \quad \Leftrightarrow \quad x(1) \geq y(1), \\
x \succcurlyeq_{2} y \quad \Leftrightarrow \quad x(2) \geq y(2), \\
x \succcurlyeq_{3} y \quad \Leftrightarrow \quad x(3) \geq y(3) .
\end{array}\right.
$$

Consider now the following problem: $\Pi=\{x, 0\}$, where the act $x=\left(\varepsilon,-1,-\varepsilon^{2}\right)$, i.e. $x(1)=\varepsilon, x(2)=-1$ and $x(3)=-\varepsilon^{2}$. Now, it is obvious that the act

\footnotetext{
${ }^{6}$ Take for instance, $p_{3}(1)=p_{3}(3)=.5$ with $\varepsilon_{1}>\varepsilon_{3}>0$.
} 
$0_{n=3}=(0,0,0)$ is optimal for individuals 2 and 3 and the act $x$ is optimal for 1. Thus, $\Pi^{*}=\{(x(1), 0,0)\}$, that is $(\varepsilon, 0,0)$. Now, by $\boldsymbol{S} \boldsymbol{M}$, the act $(\varepsilon, 0,0) \succ^{*}$ $(0,0,0)$ for each social preference and then $\mathbf{I H}$ holds.

Example 2 (Interaction Case with Global Agreement) : Suppose three individuals $\{1,2,3\}$ such that 1 and 3 have nonsingleton individual distributions, i.e.

$$
\left\{\begin{array}{l}
p_{1}(1)=\frac{1}{2}=p_{1}(2), \\
p_{3}(2)=\frac{1}{2}=p_{3}(3)
\end{array}\right.
$$

while 2 's individual distribution is $p_{2}(2)=1$. This corresponds to $S_{1}=\{1,2\}$, $S_{2}=\{2\}$ and $S_{3}=\{2,3\}$. Suppose that all utilities are given by $u_{i}(x)=x$ for $i: 1,2,3$. Then, for two acts $x, y$, we have:

$$
\left\{\begin{array}{l}
x \succcurlyeq_{1} y \quad \Leftrightarrow \quad \frac{1}{2} x(1)+\frac{1}{2} x(2) \geq \frac{1}{2} y(1)+\frac{1}{2} y(2), \\
x \succcurlyeq_{2} y \quad \Leftrightarrow x(2) \geq y(2), \\
x \succcurlyeq_{3} y \quad \Leftrightarrow \quad \frac{1}{2} x(2)+\frac{1}{2} x(3) \geq \frac{1}{2} y(2)+\frac{1}{2} y(3) .
\end{array}\right.
$$

Note that there is here a Global Network Agreement and define the social preference $\succcurlyeq *$ as:

$$
x \succcurlyeq^{*} y \Leftrightarrow \frac{1}{6} x(1)+\frac{2}{3} x(2)+\frac{1}{6} x(3) \geq \frac{1}{6} y(1)+\frac{2}{3} y(2)+\frac{1}{6} y(3) .
$$

In order to illustrate that this social preference satisfies $\mathbf{I H}$, consider any problem $\Pi$ and optimal decision rule $\Phi$. Then for any $y \in \Pi$, the optimality of $\Phi$ yields:

$$
\left\{\begin{array}{l}
\frac{1}{2} \Phi_{1}(1)+\frac{1}{2} \Phi_{2}(1) \geq \frac{1}{2} y(1)+\frac{1}{2} y(2), \\
\Phi_{2}(2) \geq y(2) \\
\Phi_{2}(2) \geq \Phi_{2}(1) \\
\Phi_{2}(2) \geq \Phi_{2}(3) \\
\frac{1}{2} \Phi_{2}(3)+\frac{1}{2} \Phi_{3}(3) \geq \frac{1}{2} y(2)+\frac{1}{2} y(3) .
\end{array}\right.
$$

Multiplying the two first and the very last inequalities by $\frac{1}{3}$, the two others by $\frac{1}{6}$ and summing up gives rise to the following equation:

$$
\frac{1}{6} \Phi_{1}(1)+\frac{2}{3} \Phi_{2}(2)+\frac{1}{6} \Phi_{3}(3) \geq \frac{1}{6} y(1)+\frac{2}{3} y(2)+\frac{1}{6} y(3) .
$$

Hence, we can conclude that $x \succcurlyeq^{*} y$ for all $x \in \Pi^{*}$. Since it works for every $y \in \Pi$, then $\mathbf{I H}$ holds.

\section{References}

Akerlof, G.A. (1997): 'Social Distance and Social Decisions,' Econometrica 65, 1005-1027. York.

Arrow, K.J. (1951): Social Choice and Individual Values. Wiley: New

Billot, A. (2003): 'How Liberalism Kills Democracy: Sen's Theorem Revisited,' Public Choice 116, 247-270.

Billot, A. and B. Walliser (1999): 'Epistemic Properties of Knowledge Hierarchy,' Journal of Mathematical Economics 32, 185-206. 
Hughes, G.E. and M.J. Cresswell (1989): An Introduction to Modal Logic. Routledge: London.

Kreps, D.M. (1988): Notes on the Theory of Choice. Westview Press: Boulder.

KrIPke, S. [1963] : 'Semantical Analysis of Modal Logic,' Z. Math Logik Grundlag der Math 9, 67-96

Luttmer, E.F.P. (2001): 'Group Loyalty and the Taste for Redistribution,' Journal of Political Economy 109, 500-528.

Morris, S. (1997): 'Interaction Games: A Unified Analysis of Incomplete Information, Local Interaction and Random Matching,' CARESS Working Paper 97-02, University of Pennsylvania.

Morris, S. (2000): 'Contagion,' Review of Economic Studies 67, 57-78.

Nozick, R. (1981): Philosophical Explanations. Clarendon: Oxford.

Postlewaite, A. (1998): 'The Social Basis of Interdependent Preferences,' European Economic Review 42, 779-800.

Rothschild, E. (1994): 'Adam Smith and the Invisible Hand,' The American Economic Review 84, 319-322.

Samet, D. and D. Schmeidler (2003): 'Between Liberalism and Democracy,' Journal of Economic Theory 110, 213-233. 